\title{
Two-Dimensional Axis Mapping Using LiDAR
}

\author{
Marc J. Gallant and Joshua A. Marshall, Senior Member, IEEE
}

\begin{abstract}
This paper introduces two-dimensional axis mapping, which estimates axis maps (AMs) based on LiDAR measurements. An AM describes the dominant orientations of surfaces in an environment, and is void of positional information. As a consequence of the directional nature of the map, there are significant differences compared to traditional mapping algorithms. Experimental results are presented from simulated, indoor, outdoor, and underground environments. One major application of AMs is for heading estimation. An example of this application is shown to effectively bound the growth of heading error in both an indoor and an outdoor environment.
\end{abstract}

Keywords—mobile robots, mapping, directional statistics, LiDAR.

\section{INTRODUCTION}

$\mathbf{T}$ HIS paper introduces two-dimensional axis mapping (2DAM), which is a new algorithm that maps the dominant axes present in an environment. In two dimensions, axes are used to represent the orientations of flat surfaces in the environment, and are parameterized as angles invariant to rotations of $\pi$. An axis map (AM) is a set of axes and is completely void of positional information. Unlike most map representations, the relationship between the environment and the entries of an AM is not injective. Put differently, the axes of many distinct physical surfaces (e.g., walls, cars, a fence, etc.) may be represented by the same entry in an AM. As a result, AMs are extremely compact, even for large environments. In fact, for semi-structured environments, an AM with only two entries may sufficiently describe all dominant axes.

Robotic mapping is a broad area of research that has many practical applications. One application for two-dimensional AMs is as part of a vehicle or robot heading estimation algorithm. By representing large environments by a succinct set of angles, a LiDAR compass (i.e., a lightweight real-time heading estimation algorithm that extracts axes from LiDAR scans; see Section V) can be used to bound the growth of heading errors. This paper demonstrates that suitable AMs can be estimated in a variety of indoor, outdoor, and underground environments, making a LiDAR compass a feasible substitute for a gyroscope, compass, or even scan matching.

Three-dimensional AMs also have practical applications. For example, in geotechnical engineering such maps are called stereonets and are used to identify structural features [1] and support stability analysis [2]. An automated and remote approach to axis mapping could greatly improve the efficiency, safety, and coverage of stereonets.

2DAM is analogous to pose-graph simultaneous localization and mapping (SLAM) constrained to the sample space of

M. J. Gallant (corresponding author) and J. A. Marshall are with the Mining Systems Laboratory, Queen's University, 25 Union Street, Kingston, ON, Canada, K7L 3N6. Email: \{m.gallant,joshua.marshall\}@queensu.ca, website: https://msl.engineering.queensu.ca directional random variables (see Section II-A). The goal is to optimize a heading-graph, whose nodes represent headings of the robot at distinct points of its trajectory, and whose edges represent the negative log-likelihood of observed rotations between nodes. The absence of positional information in AMs and the directional nature of the state produces some significant departures from the well-studied nonlinear leastsquares approach to SLAM [3], [4].

In particular, this paper develops the following facets unique to axis mapping: (i) axis extraction from 2D LiDAR scans (Section II-D); (ii) criteria for deciding when to add new nodes without positional information (Section III-A); (iii) data association and in a densely-connected graph with directional data (Section III-B); (iv) optimization of the heading-graph without violating the sample space of directional random variables (Section III-D). The main contribution of this paper is the development and testing of algorithms to efficiently and effectively meet these challenges, resulting in two-dimensional axis maps suitable for robotic mapping applications (e.g., use by a LiDAR compass).

\section{A. Organization of this Paper}

This section concludes with a survey of related work in Section I-B. Next, Section II provides background information on axes, directions, and extracting axes from LiDAR scans. Section III presents the algorithms that comprise 2DAM, and how they differ from traditional pose-graph SLAM. Finally, Section IV presents experimental results in a variety of environments and Section V demonstrates an application of AMs: heading estimation using a LiDAR compass.

\section{B. Related Work}

At its core, 2DAM is a least-squares optimization problem in the sample space of directional random variables [5]. Due to the prevalence of directional data in natural phenomena (e.g., migratory directions of animals, orientation of rock masses in geology, time of day/month/year), there is existing work on regression analysis with directional observations.

One of the first to develop a regression analysis that specifically accounts for directional data was Gould [6], who developed a technique to model the spatial orientation of vectorcardiograms (i.e., the electric activity of the heart). Gould modelled a vector of linear covariates $\mathrm{x}$ (i.e., independent variables) as the mean $\mu+\boldsymbol{\beta}^{\top} \mathbf{x}$ of a von Mises distribution [5], and solved for the maximum-likelihood estimate of the vector of parameters $\boldsymbol{\beta}$. This approach yields an infinite number of solutions (which Gould addressed with heuristics), and was later improved [7], [8] by wrapping $\boldsymbol{\beta}^{\top} \mathbf{x}$ to the unit circle. A regression technique where the independent variables $\mathrm{x}$ are also directional random variables was developed by Sarma and 
Jammalamadaka [9]. Here, the real and imaginary components of a directional observation $e^{i \theta}=\cos (\theta)+i \sin (\theta)$ are each modelled as a finite Fourier series (given an angular covariate $\alpha$ ). The Fourier coefficients are then determined to maximize the likelihood of all the observations. Lund [10] later developed a similar approach that considers the case of a combination of both linear and angular covariates. Although developed for directional random variables, these regression techniques do not address the case where the parameters being determined (e.g., $\boldsymbol{\beta}$ ) are themselves directional random variables (which is the case for 2DAM, presented in this paper).

Until very recently, directional random variables in mobile robot state estimation were most commonly assumed to be locally Gaussian. State spaces containing these variables (e.g., the orientation of a robot) are modelled as a manifold, and operations (such as the error between an observation and the model) are calculated on a local tangent plane. This approach is described in detail by Hertzberg et al. [11]. The error introduced by this approximation is negligible if the variance of the directional variables is sufficiently small. 2DAM is based partly on the manifold least-squares approach described by Hertzberg et al., with the crucial difference that the error function has a constant Jacobian. As a result, the optimal solution can be determined without numerically approximating a Jacobian or using an iterative nonlinear least squares algorithm.

Recent work has relaxed the Gaussian assumption on directional random variables by using distributions well defined on the circle. Azmani et al. [12] introduced a recursive filter using the von Mises distribution; however, a linear observation model is required. A recursive algorithm for nonlinear observations based on deterministic sampling was developed by Kurz et al. [13]. However, unlike 2DAM, these algorithms are all recursive filters (i.e., observations are considered sequentially, not simultaneously), have no data association component, and have only been tested in simulation.

Finally, the Linear Approximation for Pose-Graph Optimization (LAGO) algorithm by Carlone et al. [14], [15] involves solving the heading-graph of a two-dimensional trajectory, as is done in 2DAM. LAGO is subdivided into two independent quadratic optimization problems: first, the orientations are optimized (with special treatment given to the angle wrapping problem), and second, the solved orientations are used to optimize the positions. However, unlike LAGO, we approach this problem at both the front-end (graph construction) and back-end (graph optimization). LAGO receives as its input the constructed graph, which means that data association, loop closure detection, sensor fusion, etc., have already been performed. However, if the goal is to solve only for orientations, the graph can be augmented with heading loop closures. For example, consider two similarly-oriented yet physically distinct surfaces in the environment. If this similarity can be detected, an association can be made between the orientations of two nodes whose relative position is well outside the range of the LiDAR. This can result in a densely-connected graph even if the robot never returns to previous locations. Also, to be able to perform data association among axes for the full trajectory (dead-reckoning alone would make detecting heading loop closures difficult), 2DAM constructs the heading- graph incrementally, optimizing each time a node is added. Consequently, this gives the optimization algorithm an initial guess, resulting in a different back-end as compared to LAGO.

\section{PRELIMINARIES}

2DAM makes extensive use of axial and directional random variables, which are defined in Section II-A. Definitions of axis maps, axis observations, and the state are provided in Section II-C, and the algorithm that extracts axes from LiDAR scans is detailed in Section II-D.

\section{A. Axial and Directional Random Variables}

An entry of an axis map is an axial random variable and the heading of a robot is a directional random variable. This nomenclature taken from directional statistics [5].

Definition 1. A random variable $\Phi$ is called an axial random variable if its sample space is $[0, \pi)$.

Definition 2. A random variable $\Theta$ is called a directional random variable if its sample space is $[-\pi, \pi)$.

Neither $\Phi$ nor $\Theta$ can be exactly represented by a Gaussian distribution because of their bounded sample spaces. An appropriate distribution for axial and directional random variables is the wrapped normal (WN) distribution [5], denoted $W N\left(\theta, \sigma^{2}\right)$, which has the probability density function (PDF)

$$
f_{W N}\left(x ; \theta, \sigma^{2}\right):=\frac{1}{\sigma \sqrt{2 \pi}} \sum_{k=-\infty}^{\infty} \exp \left[\frac{-(x-\theta+n \pi k)^{2}}{2 \sigma^{2}}\right] .
$$

For axial random variables, $n=1, x \in[0, \pi)$, and $\theta \in[0, \pi)$, and for directional random variables, $n=2, x \in[-\pi, \pi)$, and $\theta \in[-\pi, \pi)$. In both cases, $\sigma \in \mathbb{R}$. Note how the WN PDF is simply an infinite series of Gaussian PDFs $f_{N}\left(x ; \theta-n \pi k, \sigma^{2}\right)$. As a result, for the same range, the probability enclosed by a WN distribution within some distance from the mean (e.g., $3 \sigma$ ) is larger than that of a Gaussian distribution in the same interval. In practice, $3 \sigma \ll \frac{\pi}{2}$ for axial random variables and $3 \sigma \ll \pi$ for directional random variables in 2DAM. As a result, the vast majority of the probability density is well-represented by the first $(k=0)$ term of a WN distribution.

Assumption 1. The axial and directional random variables used in this paper are reasonably approximated by Gaussian distributions, such that $3 \sigma \ll \frac{\pi}{2}$ and $3 \sigma \ll \pi$, respectively.

\section{B. Linear Combinations of Directional Random Variables}

Let $X \sim N\left(x, \sigma_{x}^{2}\right)$ and $Y \sim N\left(y, \sigma_{y}^{2}\right)$ be independent Gaussian random variables. If $Z=X+Y$, then $Z \sim N\left(z, \sigma_{z}^{2}\right)$, where $z=x+y$ and $\sigma_{z}^{2}=\sigma_{x}^{2}+\sigma_{y}^{2}$. However, by Definition 2, when $X$ and $Y$ are directional random variables being approximated by Gaussian distributions it must be that $z \in[-\pi, \pi)$, which is not guaranteed (e.g., consider $x=\pi$ and $y=\pi$ ). Additionally, we require $3 \sigma_{z} \ll \pi$ by Assumption 1 .

Definition 2 is satisfied by mapping a linear combination of directional random variables back to $[-\pi, \pi)$. Let $\boldsymbol{\theta}=$ $\left[\begin{array}{lll}\theta_{1} & \ldots & \theta_{n}\end{array}\right]^{\top}$, where $\theta_{i}$ is the mean of an axial or directional 
random variable, and let $\mathbf{a} \in \mathbb{R}^{n}$ such that $\mathbf{a}^{\top} \boldsymbol{\theta}+\beta$ is a linear combination of the entries of $\boldsymbol{\theta}$ offset by $\beta \in \mathbb{R}$. This result is wrapped to $[-\pi, \pi)$ by the function $f_{d}: \mathbb{R} \rightarrow[-\pi, \pi)$; i.e.,

$$
f_{d}\left(\mathbf{a}^{\top} \boldsymbol{\theta}+\beta\right)=\operatorname{atan} 2\left(\sin \left(\mathbf{a}^{\top} \boldsymbol{\theta}+\beta\right), \cos \left(\mathbf{a}^{\top} \boldsymbol{\theta}+\beta\right)\right) \text {. }
$$

Proposition 1. For all $\mathbf{a}^{\top} \boldsymbol{\theta}+\beta$, the Jacobian $\mathbf{f}$ of $f_{d}\left(\mathbf{a}^{\top} \boldsymbol{\theta}\right)$ with respect to $\boldsymbol{\theta}$ exists and is simply $\mathbf{f}=\mathbf{a}^{\top}$.

Proof: Using the chain rule, the total derivative of $f_{d}$ is

$$
\begin{aligned}
\frac{d f_{d}\left(\mathbf{a}^{\top} \boldsymbol{\theta}+\beta\right)}{d \boldsymbol{\theta}} & =\frac{\partial f_{d}\left(\mathbf{a}^{\top} \boldsymbol{\theta}+\beta\right)}{\partial \cos \left(\mathbf{a}^{\top} \boldsymbol{\theta}+\beta\right)} \frac{d \cos \left(\mathbf{a}^{\top} \boldsymbol{\theta}+\beta\right)}{d \boldsymbol{\theta}}+ \\
\frac{\partial f_{d}\left(\mathbf{a}^{\top} \boldsymbol{\theta}+\beta\right)}{\partial \sin \left(\mathbf{a}^{\top} \boldsymbol{\theta}+\beta\right)} \frac{d \sin \left(\mathbf{a}^{\top} \boldsymbol{\theta}+\beta\right)}{d \boldsymbol{\theta}} & \sin ^{2}\left(\mathbf{a}^{\top} \boldsymbol{\theta}+\beta\right)\left(\mathbf{a}^{\top}\right)+ \\
& =\mathbf{a}^{\top} \quad \cos ^{2}\left(\mathbf{a}^{\top} \boldsymbol{\theta}+\beta\right)\left(\mathbf{a}^{\top}\right)
\end{aligned}
$$

Jacobians of the form $\mathbf{a}^{\top}$ are needed for data association and to add nodes to or optimize the heading-graph.

\section{Axis Maps, Axis Observations, and the State}

Axis maps (AMs) represent the axes of surfaces in an environment expressed in the inertial coordinate frame. Let an axis map $\boldsymbol{\Phi}$ be an $m$-dimensional axial random variable with $\boldsymbol{\Phi} \sim N(\boldsymbol{\phi}, \mathbf{M})$. Axis observations are the result of transforming LiDAR scans into observations of the axes present in the environment, expressed in the robot coordinate frame. Let an axis observation $\mathbf{Z}$ be a $p$-dimensional axial random variable with $\mathbf{Z} \sim N(\mathbf{z}, \mathbf{R})$, where $\mathbf{z}:=\left[\begin{array}{lll}z^{(1)} & \ldots & z^{(p)}\end{array}\right]^{\top}$ and $\mathbf{R}:=\operatorname{diag}\left(\sigma_{z^{(1)}}^{2}, \ldots, \sigma_{z^{(p)}}^{2}\right)$. Note that surfaces with similar axes (e.g., parallel walls) are represented by one axis in $\mathbf{Z}$.

The state represents robot headings at a particular points along its trajectory, expressed in the inertial coordinate frame. Let the state $\Theta$ be an $n$-dimensional directional random variable with $\boldsymbol{\Theta} \sim N(\boldsymbol{\theta}, \mathbf{P})$, where $\boldsymbol{\theta}:=\left[\begin{array}{lll}\theta_{1} & \ldots & \theta_{n}\end{array}\right]^{\top}$. The goal of $2 \mathrm{DAM}$ is to find the optimal state mean vector $\boldsymbol{\theta}^{*}$ that maximizes the likelihood of all axis observations and gyroscope measurements. The optimal AM is then produced by rotating the axis observations at each entry in the state to the inertial coordinate frame.

\section{Axis Extraction}

Axis extraction is the process of transforming a twodimensional LiDAR scan into an axis observation. The algorithm consists of the following steps:

(i) outliers are filtered from the LiDAR data based on large range differences compared to neighbouring points;

(ii) the axis of each individual point is calculated by fitting a line to its $n$ nearest neighbours (typically $n=6$ to 12 ) using a simple Deming regression [16], which is a linear regression that accounts for uncertainty in both variables;

(iii) points with highly uncertain axes (e.g., points near corners) are discarded;

(iv) the axes of all the remaining points are clustered using DBSCAN [17], which clusters only dense concentrations of similarly-oriented axes of points (ignoring outliers and sparse orientations);

(v) the circular mean [5] and variance of each cluster are calculated, and clusters with a variance below a threshold (typically $3 \sigma_{z(j)} \ll \frac{\pi}{2}$, to coincide with Assumption 1) are returned.

The clustering of similarly-oriented point axes into a single axis is illustrated under the Axis Extraction block of Fig. 1.

\section{TwO-DIMENSIONAL AXIS MAPPING}

Two-dimensional axis mapping (2DAM) is a new algorithm used to determine the dominant orientations of an environment. It is accomplished by optimizing a heading-graph, whose nodes are headings and whose edges represent the negative log-likelihood of rotations derived from axis observations or gyroscope measurements. An overview of the 2DAM algorithm is provided in Fig. 1. The following subsections describe the details behind each of the blocks in this figure. The algorithm differs in many ways from pose-graph SLAM; these differences are highlighted throughout the text.

\section{A. Adding New Nodes to the Heading-Graph}

A gyroscope can be used to estimate the rotation between sequential nodes in the heading-graph. Gyroscope measurements are integrated to produce the directional random variable $\Delta \Theta \sim N\left(\delta \theta, \sigma_{\delta \theta}^{2}\right)$. An initial estimate of the mean heading of the new node is calculated by using the motion model $g: \mathbb{R} \rightarrow[-\pi, \pi)$; i.e.,

$$
g(\boldsymbol{\theta}, \delta \theta)=f_{d}\left(\theta_{n}+\delta \theta\right) .
$$

The covariance matrix $\mathbf{P}$ must also be augmented to include the new node. By Proposition 1, the Jacobian $\mathbf{g}^{\top}$ of $g$ with respect to $\boldsymbol{\theta}$ is $\left[\begin{array}{llll}0_{1} & 0_{2} & \ldots & 1_{n}\end{array}\right]$. The new state mean vector and covariance matrix are then

$$
\begin{aligned}
& \boldsymbol{\theta} \leftarrow\left[\begin{array}{llll}
\theta_{1} & \ldots & \theta_{n} & g(\boldsymbol{\theta}, \delta \theta)
\end{array}\right]^{\top}, \\
& \mathbf{P} \leftarrow\left[\begin{array}{cc}
\mathbf{P} & \mathbf{P g} \\
\mathbf{g}^{\top} \mathbf{P} & \mathbf{g}^{\top} \mathbf{P g}+\sigma_{\delta \theta}^{2}
\end{array}\right] .
\end{aligned}
$$

This augmentation of the state is illustrated under the Add New Node block in Fig. 1.

The decision to add a new node in pose-graph SLAM is commonly triggered once the estimated change in position or orientation of the robot exceeds a threshold (e.g., $0.5 \mathrm{~m}$ or $0.5 \mathrm{rad}$, respectively) [3]. This ensures there is sufficient overlap in observations between sequential nodes. However, due to the lack of positional information, triggering new nodes is less straightforward for heading-graphs. As a result, a new node is triggered if any of the following conditions are met:

(i) $\delta \theta$ is greater than a threshold (typically $10^{\circ}-25^{\circ}$ );

(ii) $\sigma_{\delta \theta}^{2}$ is greater than a threshold (to meet Assumption 1);

(iii) A timeout occurs (typically 5-25 s) and the circular mean of all point axes has changed by a threshold (typically $\left.8^{\circ}-15^{\circ}\right)$

These three conditions (illustrated under the New Node Triggered block in Fig. 1) ensure that nodes are added less frequently in areas with constant axes (e.g., corridors), and more frequently while the robot is rotating. 


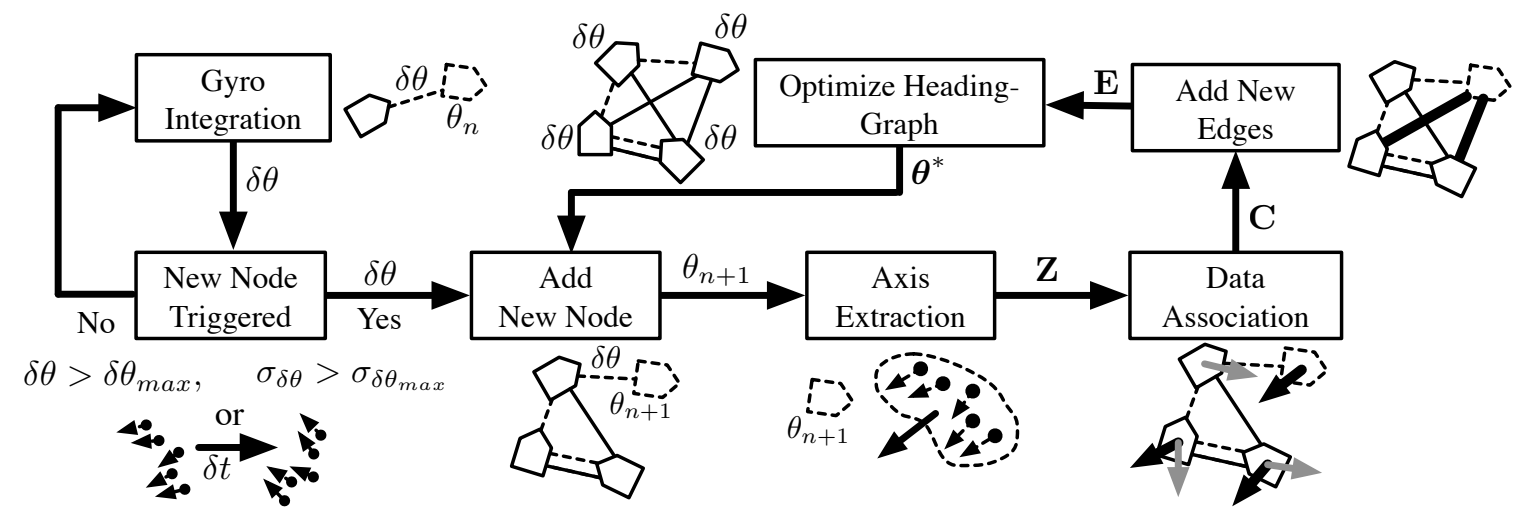

Fig. 1. Overview of the 2DAM algorithm. A gyroscope is integrated until a new node is triggered. A new node is added to the heading-graph using the gyroscope integration (Section III-A), at which point the axes of the environment are extracted from a LiDAR scan (Section II-D). The extracted axes are associated with the axes of all the other nodes (Section III-B), and successful associations are transformed into new edges in the heading-graph (Section III-C). The graph is then optimized before a new node is added (Section III-D).

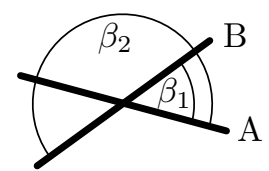

Fig. 2. The rotation ambiguity between axes $\mathrm{A}$ and $\mathrm{B}$. Because axes are invariant to rotations of $\pm \pi$, axis $\mathrm{A}$ can be aligned with axis $\mathrm{B}$ through the equivalent rotations $\beta_{1}$ and $\beta_{2}$. If $\beta_{1}$ is always taken as the smaller absolute angle, then $\beta_{1} \in\left[-\frac{\pi}{2}, \frac{\pi}{2}\right)$ and $\beta_{2} \in\left[\frac{\pi}{2}, \frac{3 \pi}{2}\right)$.

\section{B. Data Association}

Data association uses the joint compatibility branch and bound (JCBB) algorithm [18] to associate axis observations between nodes. JCBB finds the largest jointly compatible injective subset of correspondences between axis observations. However, data association using heading-graphs has two important differences compared to pose-graphs. First, correspondence checks cannot be restricted to nearby nodes because axis observations are not injective. Put differently, two physical surfaces in the environment that are positioned far apart are considered the same "landmark" (in the pose-graph sense) if they share a similar axis. Second, calculating the Mahalanobis distance between axis observations requires an additional step to resolve the $\pm \pi$ rotation ambiguity (illustrated in Fig. 2).

This section describes how the Mahalanobis distance between to axis observations is calculated. This method is then used to perform the full JCBB algorithm. Let $z_{i}^{(a)}$ and $z_{j}^{(b)}$ be the $a$-th and $b$-th mean axis observations at the $i$-th and $j$-th nodes, respectively. The error $v_{i, j}^{(a, b)} \in\left[-\frac{\pi}{2}, \frac{\pi}{2}\right)$ between these two observations is calculated by first rotating them to the inertial frame, and then selecting the smaller of the two rotations between them (see Fig. 2); i.e.,

$$
v_{i, j}^{(a, b)}=f_{d}\left(\left(z_{j}^{(b)}+\theta_{j}\right)-\left(z_{i}^{(a)}+\theta_{i}\right)\right),
$$

where $v_{i, j}^{(a, b)} \longleftarrow f_{d}\left(v_{i, j}^{(a, b)}+\pi\right)$ if (5) yields $v_{i, j}^{(a, b)} \notin$ $\left[-\frac{\pi}{2}, \frac{\pi}{2}\right)$. The variance of the error $\sigma_{v_{i, j}^{(a, b)}}^{2}$ is

$$
\sigma_{v_{i, j}^{(a, b)}}^{2}=\sigma_{z_{i}^{(a)}}^{2}+\sigma_{z_{j}^{(b)}}^{2}+\mathbf{v}_{i, j}^{\top} \mathbf{P} \mathbf{v}_{i, j},
$$

where by Proposition 1 , the Jacobian $\mathbf{v}_{i, j}^{\top}$ of $v_{i, j}^{(a, b)}$ with respect to $\boldsymbol{\theta}$ is $\mathbf{v}_{i, j}^{\top}=\left[\begin{array}{lllllll}0_{1} & \ldots & -1_{i} & \ldots & 1_{j} & \ldots & 0_{n}\end{array}\right]$. The Mahalanobis distance $m_{i, j}^{(a, b)} \in \mathbb{R}^{+}$is then

$$
m_{i, j}^{(a, b)}=\sqrt{\frac{\left(v_{i, j}^{(a, b)}\right)^{2}}{\sigma_{v_{i, j}^{(a, b)}}^{2}}} .
$$

When a new node is added to the heading-graph, the Mahalanobis distance is calculated between its axis observations and the axis observations of all the other nodes. Note that the relatively small number of axis observations at each node (typically $2-4$ observations) makes this computationally tractable. JCBB then produces a set of correspondences between the nodes, which are stored in a compatibility matrix C. The illustration under the Data Association block in Fig. 1 highlights associations with axis observations of the new node.

\section{Adding New Edges}

An edge in the heading-graph represents the negative loglikelihood of an observed rotation between the nodes it connects. Let an observed rotation $R_{i, j}$ from the $i$-th node to the $j$-th node be a directional random variable with $R_{i, j} \sim$ $N\left(r_{i, j}, \sigma_{r_{i, j}}^{2}\right)$. There are two sources of observed rotations between nodes: gyroscope measurements and associated axis observations in C. Observed rotations from gyroscope measurements occur between sequential nodes and have the form

$$
r_{i, j}^{(\delta \theta)}=\delta \theta, \quad \sigma_{r_{i, j}^{(\delta \theta)}}^{2}=\sigma_{\delta \theta}^{2} .
$$

There is also an observed rotation for each association in C. Let an entry of $\mathbf{C}$ be the $a$-th and $b$-th axis observations 
of the $i$-th and $j$-th nodes, respectively; then

$$
\begin{aligned}
r_{i, j}^{(a, b)} & = \begin{cases}f_{d}\left(z_{i}^{(b)}-z_{j}^{(a)}\right), & \text { if } v_{i, j}^{(a, b)} \in\left[-\frac{\pi}{2}, \frac{\pi}{2}\right) \\
f_{d}\left(z_{i}^{(b)}-z_{j}^{(a)}+\pi\right), & \text { otherwise. }\end{cases} \\
\sigma_{r_{i, j}^{(a, b)}}^{2} & =\sigma_{z_{i}^{(a)}}^{2}+\sigma_{z_{j}^{(b)}}^{2} .
\end{aligned}
$$

These two cases are necessary to resolve the ambiguity illustrated in Fig. 2.

The error $e_{i, j}^{(k)}(\boldsymbol{\theta}) \in[-\pi, \pi)$ of the $k$-th observed rotation between the $i$-th and $j$-th node is

$$
e_{i, j}^{(k)}(\boldsymbol{\theta})=f_{d}\left(r_{i, j}^{(k)}-h_{i, j}(\boldsymbol{\theta})\right),
$$

where $h_{i, j}: \mathbb{R} \rightarrow[-\pi, \pi)$ is the predicted rotation

$$
h_{i, j}(\boldsymbol{\theta})=f_{d}\left(\theta_{j}-\theta_{i}\right) .
$$

Let $\mathbf{E}=\left\{(i, j, k)_{1}, \ldots,(i, j, k)_{e}\right\}$, where $(i, j, k)$ represents the $k$-th observed rotation between the $i$-th and $j$-th node. The negative log-likelihood $\ell_{i, j}^{(k)}$ of an edge in $\mathbf{E}$ is then

$$
\ell_{i, j}^{(k)}=\frac{\left(e_{i, j}^{(k)}(\boldsymbol{\theta})\right)^{2}}{\sigma_{r_{i, j}^{(k)}}^{2}} .
$$

The illustration beside the Add New Edges block in Fig. 1 shows new edges added to the heading-graph based on newly associated axes in $\mathbf{C}$.

\section{Optimizing the Heading-Graph}

The heading-graph is optimized each time a new node is added. This consists of finding the optimal state mean vector $\boldsymbol{\theta}^{*}$ that minimizes the sum of the negative log-likelihood of all of the observed rotations between nodes; i.e.,

$$
\boldsymbol{\theta}^{*}=\underset{\boldsymbol{\theta}}{\operatorname{argmin}} \sum_{(i, j, k) \in \mathbf{E}} \ell_{i, j}^{(k)}
$$

Because $\boldsymbol{\Theta}$ is a vector of directional random variables, $\boldsymbol{\theta}^{*}$ cannot be determined directly (e.g., if $r_{i, j}^{(k)}=2^{\circ}$, one must consider the possibility that $\theta_{j}=-179^{\circ}$ and $\theta_{i}=179^{\circ}$ ). Different methods for solving nonlinear least squares problems have been extensively explored for pose-graphs; e.g., GaussNewton [19], stochastic gradient descent [20], and LevenbergMarquardt [21]. These methods approximate $e_{i, j}^{(k)}(\boldsymbol{\theta})$ by a firstorder Taylor series expansion and iteratively solve for the optimal perturbation to the state until convergence. However, unlike pose-graph SLAM, the perturbed state is exactly represented by its first-order Taylor series expansion, resulting in a closed-form solution to the optimization problem.

Proposition 2. Given an initial estimate $\overline{\boldsymbol{\theta}}$ of the state mean, the negative log-likelihood $\ell_{i, j}^{(k)}$ of each edge in $\mathbf{E}$ is quadratic with respect to a perturbation $\boldsymbol{\delta} \boldsymbol{\theta}$ of the state mean.
Proof: The Taylor-series expansion of $e_{i, j}^{(k)}$ evaluated at the initial guess $\overline{\boldsymbol{\theta}}$ is

$$
\begin{aligned}
e_{i, j}^{(k)}(\overline{\boldsymbol{\theta}}+\boldsymbol{\delta} \boldsymbol{\theta})=\left.e_{i, j}^{(k)}\right|_{\overline{\boldsymbol{\theta}}}+ & \left(\left.\frac{\partial e_{i, j}^{(k)}}{\partial \boldsymbol{\theta}}\right|_{\overline{\boldsymbol{\theta}}}\right) \boldsymbol{\delta} \boldsymbol{\theta}+ \\
& \frac{1}{2} \boldsymbol{\delta}^{\top}\left(\left.\frac{\partial^{2} e_{i, j}^{(k)}}{\partial \boldsymbol{\theta}^{2}}\right|_{\overline{\boldsymbol{\theta}}}\right) \boldsymbol{\delta} \boldsymbol{\theta}+\ldots
\end{aligned}
$$

By Proposition 1, the partial derivatives are

$$
\begin{aligned}
& \left.\frac{\partial e_{i, j}^{(k)}}{\partial \boldsymbol{\theta}}\right|_{\overline{\boldsymbol{\theta}}}=\left[\begin{array}{lllllll}
0_{1} & \ldots & 1_{i} & \ldots & -1_{j} & \ldots & 0_{n}
\end{array}\right] \\
& =\mathbf{e}_{i, j}^{\top} \\
& \left.\frac{\partial^{m} e_{i, j}^{(k)}}{\partial \boldsymbol{\theta}^{m}}\right|_{\overline{\boldsymbol{\theta}}}=\mathbf{0}^{\top} \quad \text { for } m \geq 2 .
\end{aligned}
$$

Substituting (15) into (13) yields

$$
\ell_{i, j}^{(k)}=\frac{\left(e_{i, j}^{(k)}+\mathbf{e}_{i, j}^{\top} \boldsymbol{\delta} \boldsymbol{\theta}\right)^{2}}{\sigma_{r_{i, j}^{(k)}}^{2}} .
$$

Substituting (17) into (14) transforms the optimization problem into the minimization of a quadratic function; i.e.,

$$
\boldsymbol{\delta} \boldsymbol{\theta}^{*}=\underset{\boldsymbol{\delta} \boldsymbol{\theta}}{\operatorname{argmin}} \sum_{(i, j, k) \in \mathbf{E}} \frac{\left(e_{i, j}^{(k)}+\mathbf{e}_{i, j}^{\top} \boldsymbol{\delta} \boldsymbol{\theta}\right)^{2}}{\sigma_{r_{i, j}^{(k)}}^{2}},
$$

where the optimal state mean vector $\boldsymbol{\theta}^{*}$ is calculated by perturbing the initial estimate by the optimal perturbation $\delta \boldsymbol{\theta}^{*}$; i.e., $\boldsymbol{\theta}^{*}=f_{d}\left(\overline{\boldsymbol{\theta}}+\boldsymbol{\delta} \boldsymbol{\theta}^{*}\right)$. In this case, the wrapping function $f_{d}$ is applied separately to each entry of its argument. The standard gradient-vector minimization technique is used to solve (18), yielding

$$
\boldsymbol{\delta} \boldsymbol{\theta}^{*}=\left(\sum_{(i, j, k) \in \mathbf{E}} \frac{\mathbf{e}_{i, j} \mathbf{e}_{i, j}^{\top}}{\sigma_{r_{i, j}^{(k)}}^{2}}\right)^{-1} \sum_{(i, j, k) \in \mathbf{E}} \frac{e_{i, j}^{(k)}}{\sigma_{r_{i, j}^{(k)}}^{2}} \mathbf{e}_{i, j} .
$$

Optimization by perturbation is illustrated beside the Optimize Heading-Graph block in Fig. 1. By performing the optimization each time a new node is added, excessive dead-reckoning error is avoided that could cause erroneous associations between axes extracted at older and newer nodes.

\section{E. Generating Axis Maps}

An axis map is produced by rotating the axis observations at each node in the heading-graph to the inertial coordinate frame. Rotating the $a$-th axis extracted at the $i$-th node produces the AM entry $\phi_{i}^{(a)} \in[0, \pi)$ by

$$
\phi_{i}^{(a)}= \begin{cases}\theta_{i}+z_{i}^{(a)}, & \text { if }\left(\theta_{i}+z_{i}^{(a)}\right) \in[0, \pi) \\ \frac{1}{2} f_{d}\left(2 \theta_{i}+2 z_{i}^{(a)}\right), & \text { otherwise. }\end{cases}
$$




\section{EXPERIMENTAL RESULTS}

The 2DAM algorithm was tested using data collected from four distinct environments: (i) in simulation using a randomly generated jagged wall; (ii) indoors at Beamish-Munro Hall, a building at Queen's University; (iii) outdoors on the Queen's University campus; (iv) underground in a mining drift at the NORCAT Underground Centre. This section discusses the effectiveness of 2DAM in these environments.

\section{A. Simulation}

A simulator was constructed to test 2DAM in challenging environments, such as the jagged wall in Fig. 3a. It also allows testing in environments where the angle of a dominant axis varies among different surfaces, which is a better representation of naturally occurring surfaces (e.g., rock). The simulated LiDAR was modelled as a Hokuyo URG-04LX (same range, resolution, noise, and frequency), and the gyroscope was modelled with noise comparable to a Microstrain 3DMGX3-25 IMU. The simulated robot was driven at $0.5 \mathrm{~m} / \mathrm{s}$ while following the shape of the wall, which was randomly constructed using the algorithm described in Fig. 4. The wall illustrated in Fig. 3a consists of 200 segments, of which 169 were drawn from a eight-entry $\mathrm{AM}$, and 31 are random axes drawn from $[0, \pi)$. The resulting distributions of the non-outliers are illustrated in Fig. 3c. The estimated state, unclustered AM, and clustered AM resulting from 2DAM are shown in Figs. 3b, 3d, and 3e, respectively.

The error bars in Fig. $3 \mathrm{~b}$ represent the $3 \sigma$ uncertainty of the estimated heading at each node, which encompasses the ground truth (dashed line) at every node. Despite the absence of loop closures (in the SLAM sense), the uncertainty is evenly distributed among the nodes. In other words, unlike posegraph SLAM, heading loop closures routinely occur upon reobservation of similarly oriented surfaces, regardless of their relative position. This creates a dense heading-graph whose Hessian matrix does not contain the distinctive "arrowhead" structure of pose-graph SLAM. The highly-interconnected heading-graph reduces the likelihood of a string of "weakly" connected nodes that are attached to an un-looped graph via relatively few edges. However, one downside is the inability to use sparse methods to invert the Hessian matrix.

It is clear by comparing Figs. $3 \mathrm{c}$ and $3 \mathrm{e}$ that the estimated $\mathrm{AM}$ is an accurate representation of axes in the simulated wall. In general, the estimated axes tended to have a similar means with slightly inflated uncertainties. This inflation comes from two sources: the inherent uncertainty introduced by the noisy LiDAR and gyroscope, and the occurrence of outliers close enough to the true axes to be included by the clustering algorithm. Some of these outliers are visible in Fig. 3d, especially around the cluster near $60^{\circ}$.

\section{B. Beamish-Munro Hall}

Data was collected on the ground floor of Beamish-Munro Hall (BMH), a building at Queen's University (Kingston, ON, Canada). A blueprint of the test area is shown in Fig. 5a. A Clearpath Robotics Husky A200 mobile robot was equipped with a SICK LMS1112D laser scanner (field of view of $270^{\circ}$, resolution $0.5^{\circ}$, range $20 \mathrm{~m}$ ) oriented to scan in the horizontal plane, and a Microstrain 3DM-GX3-25 IMU, used solely for one of its gyroscopes. At the time of the experiments, the environment had a considerable amount of pedestrian traffic.

The robot was driven at speeds varying from $0.1-1.0 \mathrm{~m} / \mathrm{s}$ approximately along the path in Fig. 5a. AMs generated with 2DAM are illustrated in Figs. $5 b$ and $5 c$, respectively. The mean axes of the dominant surfaces were estimated to be (shown with standard deviations) $\phi=\left(0.7 \pm 3.4^{\circ}, 90.1 \pm 2.8^{\circ}\right)$, which is quite similar to the expected AM of $\phi=\left(0^{\circ}, 90^{\circ}\right)$ obtained by observing the blueprint in Fig. 5a.

Comparing Figs. 5b and 5c reveals that two smaller clusters appear to be joined together, increasing their uncertainty. It was determined that part of the environment with gradually curved surfaces momentarily caused incorrect data associations, leading to the addition of false edges to the heading-graph. This demonstrates a weakness of using DBSCAN: small changes of the axes of individual points along a curved surface tend to be clustered together, even if the maximum circular distance between two points in the cluster is quite large.

Although the path of the robot was approximately $130 \mathrm{~m}$ in length, the heading-graph consisted of only 46 nodes (a mean distance of $2.8 \mathrm{~m}$ between nodes). As expected based on the new node triggers described in Section III-A, relatively few nodes were added in long corridors compared to areas where the geometry of the environment required substantial heading changes or the axes of surfaces rapidly changed.

\section{Queen's University Campus}

Data was collected by driving a Taylor-Dunn SS-534 electric vehicle outdoors around Queen's University campus, along the $1.3 \mathrm{~km}$ route illustrated in Fig. 6b. The vehicle (pictured in Fig. 6a) was equipped with the same LiDAR and IMU used in the BMH experiments. The environment contained many trees, buildings, cars, pedestrians, and gardens. An additional experiment was performed using a magnetometer for heading estimation to compare with 2DAM in an outdoor environment.

The AM illustrated in Fig. 6c shows a greater variety of extracted axes compared to the indoor data at $\mathrm{BMH}$. The axes in the less dense parts of the AM are primarily attributed to two sources: environmental anomalies (e.g., exterior walls of buildings, the sides of parked cars, infrastructure around pathways, all which had flat surfaces at a variety of angles) and false positive axis extractions (i.e., a sufficiently large number of points from different parts of the LiDAR scan having similar axes and being clustered together). Although the environmental anomalies are expected (and should) appear in the AM, one way to reduce false positive axis extractions could be to cluster points based on both their axes and relative positions. Despite the ideal conditions for magnetometer-based heading estimation, the AM it generated (Fig. 6e) was much less precise than its 2DAM counterpart.

Despite the variety of extracted axes, Fig. $6 \mathrm{~d}$ shows that two dense clusters at $\phi=\left(1.3 \pm 2.5^{\circ}, 91.9 \pm 2.6^{\circ}\right)$ could be extracted. Based on the orientation of the buildings in Fig. 6b, a reasonable expected $\mathrm{AM}$ is $\phi=\left(0^{\circ}, 90^{\circ}\right)$. Once again, areas 


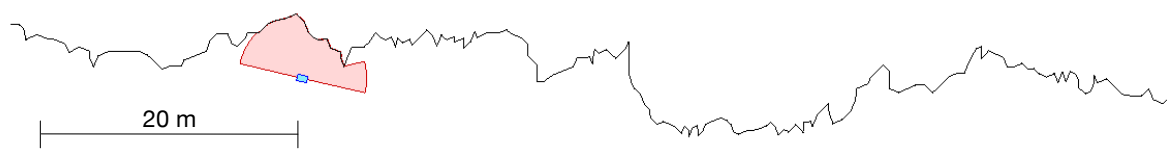

(a)

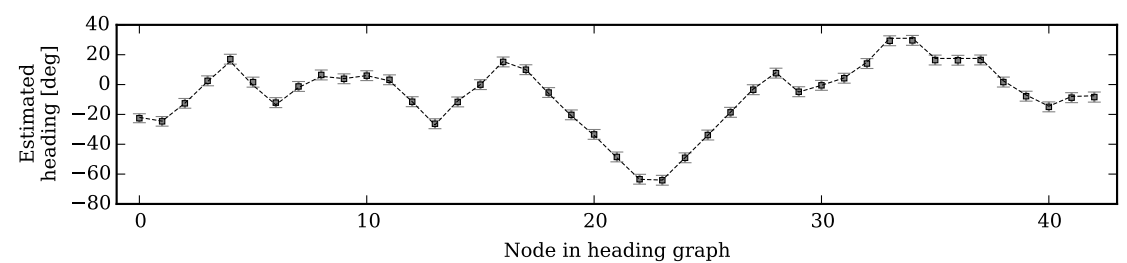

(b)

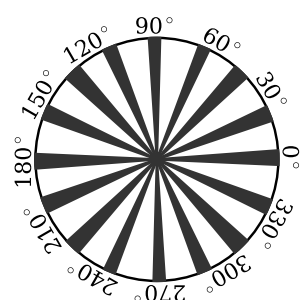

(c)

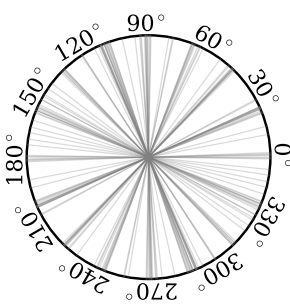

(d)

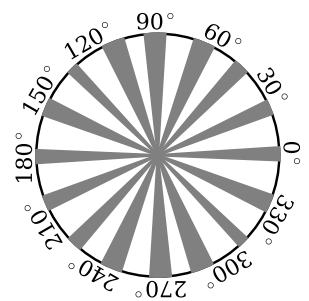

(e)

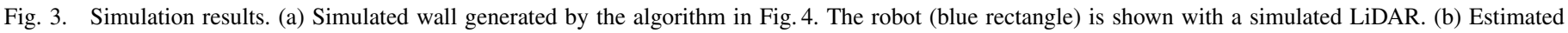

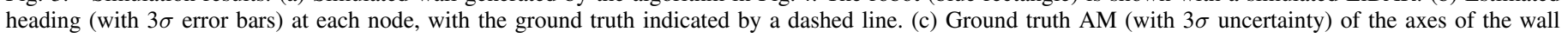
segments that are not outliers. (d) Unclustered AM. (e) AM (with $3 \sigma$ uncertainty) generated by clustering the axes in (d).

Require: $N, L_{\min }, L_{\max },\left(\mu_{1} \pm \sigma_{1}, \ldots, \mu_{M} \pm \sigma_{M}\right), P_{\text {out }}$ while \# lines $<N$ do

$L=$ sample_uniform $\left(L_{\min }, L_{\max }\right)$

Choose axis or outlier based on $P_{\text {out }}$

if axis then

Choose $\mu \pm \sigma$ from $\left(\mu_{1} \pm \sigma_{1}, \ldots, \mu_{M} \pm \sigma_{M}\right)$

$\theta=$ sample_gaussian $(\mu, \sigma) \bmod \pi$

else

$$
\theta=\text { sample_uniform }(0, \pi)
$$

end if

Append line $(L, \theta)$ to end of previous line end while

Find sample distributions from lines generated by $\left(\mu_{i}, \sigma_{i}\right)$

Fig. 4. Pseudocode for the algorithm used to generate the simulated wall in Fig. 3. The number of lines was $N=200$, the minimum and maximum line lengths were $L_{\min }=0.4 \mathrm{~m}$ and $L_{\max }=1.0 \mathrm{~m}$, respectively, and the percentage of outliers was $P_{\text {out }}=20$. The resulting eight sample distributions (shown in Fig. 3c) were $\left(0.9^{\circ} \pm 1.1^{\circ}, 22.2^{\circ} \pm 1.2^{\circ}, 46.1^{\circ} \pm 1.2^{\circ}, 67.1^{\circ} \pm\right.$ $\left.0.9^{\circ}, 91.1^{\circ} \pm 0.9^{\circ}, 113.1^{\circ} \pm 1.4^{\circ}, 134.0^{\circ} \pm 1.4^{\circ}, 157.1^{\circ} \pm 1.1^{\circ}\right)$.

with constant-axis surfaces and straight pathways resulted in a sparse heading-graph, this time averaging nearly $12 \mathrm{~m}$ between nodes. Unlike pose-graph SLAM, this distance is permitted because overlap between LiDAR scans is unnecessary. Note that relaxing the minimum number of axes in a cluster in Fig. 6c introduces another "dominant" surface at approximately $45^{\circ}$. It is speculated that chamfered corners on some buildings contributed to this cluster.

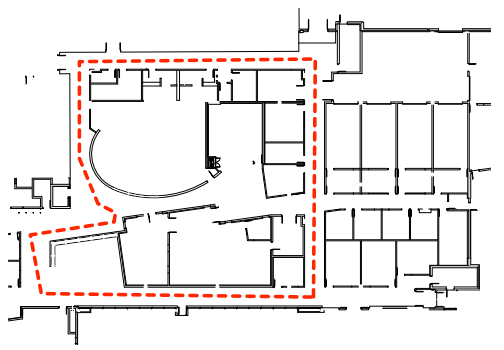

(a)

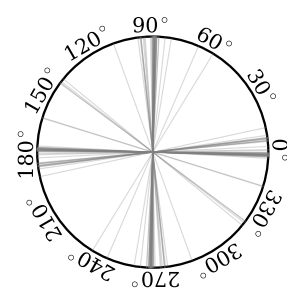

(b)

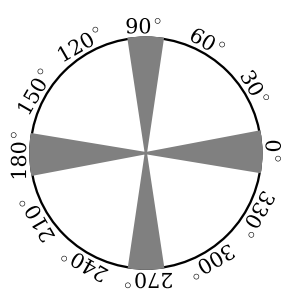

(c)
Fig. 5. Results of the Beamish-Munro Hall experiments. (a) Blueprint of the ground floor (roughly $35 \mathrm{~m} \times 55 \mathrm{~m}$ ) with a dashed line indicating the approximate path of the robot. (b) AM generated by 2DAM. (c) AM in (b) after applying DBSCAN. The width of the wedges correspond to $3 \sigma$ uncertainty.

\section{NORCAT Underground Centre}

The simulation results in Section IV-A showed that 2DAM can be applied to less structured environments with inherent uncertainty in the AM entries. One such environment is 


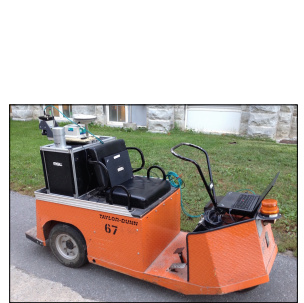

(a)

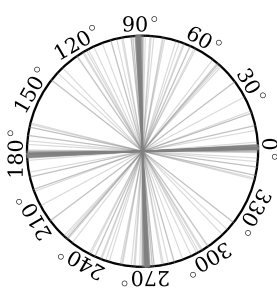

(c)

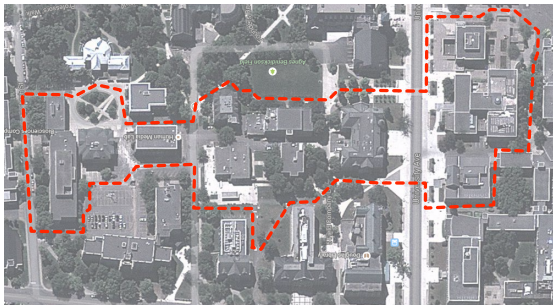

(b)

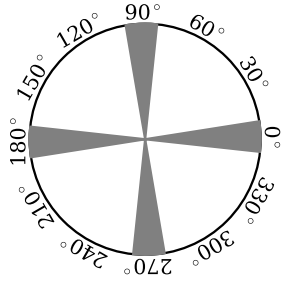

(d)

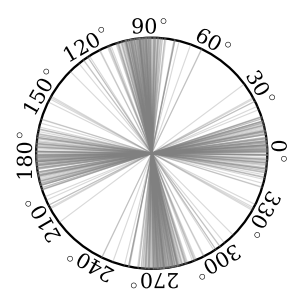

(e)
Fig. 6. Results of the Queen's campus experiments. (a) Electric vehicle used to collect data. (b) Satellite imagery of part of the campus (c)2014 DigitalGlobe), showing the dashed path driven by the vehicle. (c) AM generated by 2DAM. (d) AM in (c) after applying DBSCAN. The width of the wedges correspond to $3 \sigma$ uncertainty. (e) AM generated using a magnetometer.

underground tunnels, where exposed rock faces provide a small number of reoccurring surface orientations. Rock masses have highly anisotropic planes of weakness (or discontinuities) caused by tectonic activity, heating/cooling events, or sudden changes in stress. Discontinuities appear as groups of planes (a joint set) that have similar three-dimensional axes. In general, a limited number of joint sets (typically $<5$ ) are visible in a rock face, each distributed in orientation and spacing. Thus, joint sets should appear as clusters of axes in 2DAM.

The same robot (and sensors) used in the BMH experiments (see Section IV-B) was driven approximately $50 \mathrm{~m}$ in an underground mine tunnel at the NORCAT Underground Centre (Onaping, ON, Canada). Although pictured at a different part of the mine, Fig. 7a shows the robot and the typical structure of a rock face containing the joint sets. The robot drove over uneven ground at approximately $0.5 \mathrm{~m} / \mathrm{s}$, not necessarily in a straight line. The AMs of the drift walls are illustrated in Figs. $7 \mathrm{~b}$ and $7 \mathrm{c}$.

The clustered AM in Fig. 7c suggests that three different joint sets were prevalent in the mine drift. Ground truth was not available for this experiment, and visual inspection of the rock faces was difficult due to darkness. However, the goal of detecting distinguishable joint sets was successful. It is conceivable that a LiDAR compass (Section V) could be used in an underground mine, especially if the entries in the clustered AM could be expressed in the coordinate system of the mine. However, as discussed in Section I, the true value of this experiment is as a feasibility study for mobile 3D axis mapping. 3D axis maps of geological surfaces (called stereonets) provide measurable properties from which engineering or geological information can be inferred.

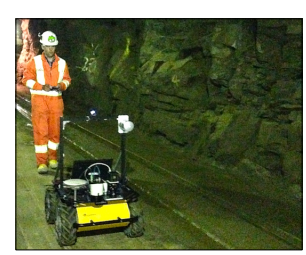

(a)

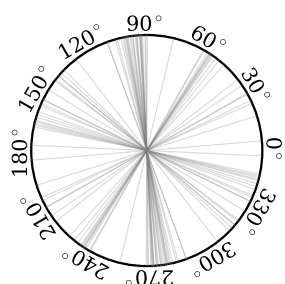

(b)

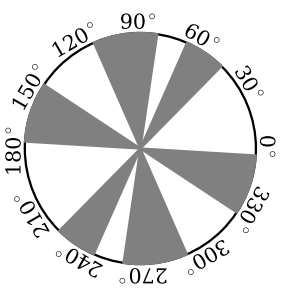

(c)
Fig. 7. Results of the NORCAT Underground Centre experiments. (a) Robot operating in the underground mine. Note the structure of the rock face on the right. (b) Unclustered AM, showing that axes extracted from the rock face are not uniformly distributed but rather form clusters around the joint set axes. (c) AM formed by clustering the axes in (b).

\section{APPLICATION: LIDAR COMPASS}

A LiDAR compass (LC) is a heading estimation algorithm that uses LiDAR and an a priori AM. Not unlike a compass or sun sensor, absolute heading information (with respect to the initial heading) can be inferred by observing entries in an AM. When paired with a sensor capable of accurately measuring translation (e.g., wheel encoders), an LC can be used to aid localization. Using an LC in this way is meant as an extremely lightweight alternative to more complex algorithms (e.g., scan matching), suitable for computationally-constrained platforms and also to provide initial pose estimates for mapping algorithms (e.g., pose-graph SLAM). A preliminary form of an LC [22] showed its effectiveness in a warehouse environment.

The steps below describe the implementation of a LiDAR compass as part of a localization algorithm; specifically, how the pose of a robot is updated given an a priori $\mathrm{AM} \boldsymbol{\Phi}$, an axis observation $\mathbf{Z}$ (see Section II-C), and an estimate of the change in position $\Delta d \in \mathbb{R}$ and in heading $\Delta \theta \in[-\pi, \pi)$ reported by wheel encoders. The state $\boldsymbol{\Theta}=(x, y, \theta, \hat{\mathbf{\Phi}})$ consists of the pose of the robot $(x, y, \theta) \in\left(\mathbb{R}^{2} \times[-\pi, \pi)\right)$ and a local AM $\hat{\boldsymbol{\Phi}}$. The entries of $\hat{\boldsymbol{\Phi}}$ consist of axes in the environment that are locally present but not in $\boldsymbol{\Phi}$. Entries are added to and removed from $\hat{\boldsymbol{\Phi}}$ based on how frequently they are observed. The purpose of $\hat{\boldsymbol{\Phi}}$ is to provide robustness to heading estimation in areas where the entries of $\hat{\boldsymbol{\Phi}}$ are less frequently observed.

1) Given $\boldsymbol{\Theta}_{k-1}$, calculate the a priori estimate $\boldsymbol{\Theta}_{k}^{-}$by passing $\Delta d$ and $\Delta \theta$ through the (nonlinear) motion model using an unscented transformation.

2) Perform data association among the axes in $\mathbf{\Phi}$ and $\mathbf{Z}$.

3) Calculate the a posteriori estimate $\Theta_{k}^{+}$using the correction step of a Kalman filter. The observation model is simply $\hat{z}=f_{d}(\phi-\theta)$, where $\phi$ is an entry of $\boldsymbol{\Phi}$. The innovation is simply $f_{d}(z-\hat{z})$, where $z$ is an entry of $\mathbf{Z}$.

4) Perform data association among the axes in $\hat{\Phi}$ and the axes in $\mathbf{Z}$ that were not associated with $\boldsymbol{\Phi}$.

5) Perform a second correction step to calculate a new $a$ posteriori estimate $\boldsymbol{\Theta}_{k}^{+}$using the new associations.

6) Add unassociated axes in $\mathbf{Z}$ to $\hat{\boldsymbol{\Phi}}$.

7) Merge similar axes; remove rarely observed axes from $\hat{\boldsymbol{\Phi}}$. Additional data sets from Beamish-Munro Hall and the 


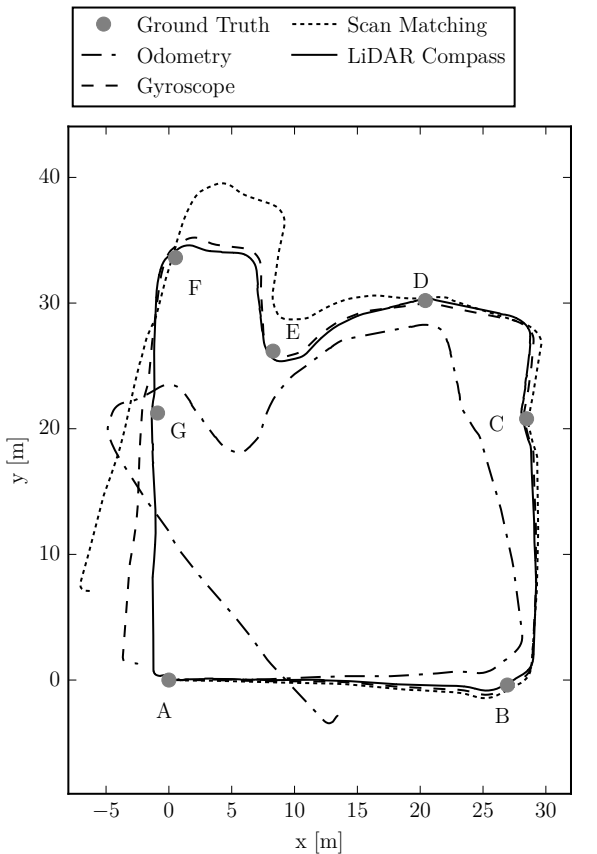

Fig. 8. The estimated paths of the robot for the BMH data set. The lettered markers indicate known ground truth positions; the ideal path should pass through all the markers. The path both started and ended at "A".

Queen's University campus were collected to test using AMs with a LiDAR compass, with the results in Sections IV-B (Fig. 5c) and IV-C (Fig. 6d) providing the a priori AMs.

\section{A. Beamish-Munro Hall}

The robot was localized using wheel encoders and an LC, gyroscope, or scan matching (using the same setup described in Section IV-B). An open source implementation [23] of the popular point-to-line scan matching algorithm by Censi [24] was used. For the LC, the a priori AM $\phi=\left(0.7 \pm 3.4^{\circ}, 90.1 \pm 2.8^{\circ}\right)$ illustrated in Fig. 5c was used. Ground truth was calculated at distinct poses (labelled A through $\mathrm{G}$ ). The robot was briefly stopped at ground truth markers to record the poses estimated by the localization algorithms.

The estimated paths of the robot and the position of the ground truth markers are shown in Fig. 8. To analyze the effectiveness of an LC for heading estimation, the heading errors recorded at each of the ground truth markers are shown in Fig. 9. It was found that the heading error is bounded by the LC when given the a priori AM produced in this paper. However, recall from Section IV-B that the uncertainty in the AM was increased due to the curved surfaces in the environment causing clustering issues. Heading estimation by an LC in these areas was affected by similar issues, causing the jumps in heading error at markers "D" and "E" in Fig. 9, although the LC was able to quickly recover.

\section{B. Queen's University Campus}

In addition to the LiDAR and IMU, the vehicle pictured in Fig. 6a was equipped with US Digital A2 encoders on the drive
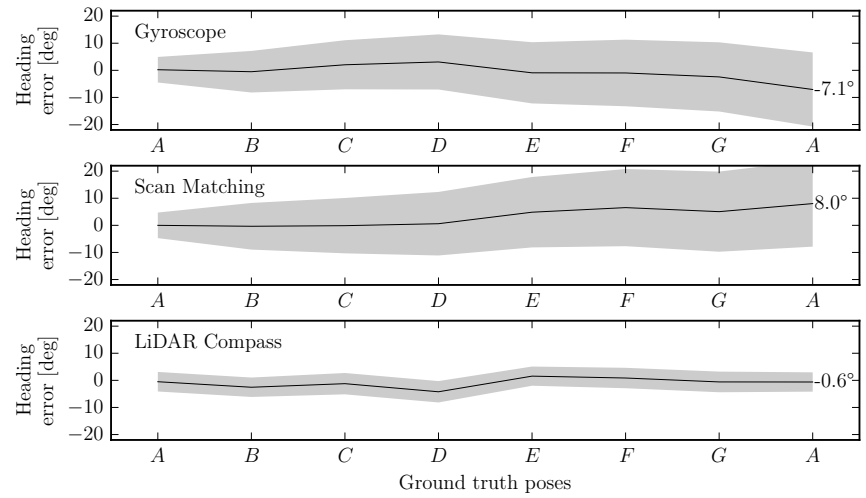

Fig. 9. The gyroscope, scan matching, and LC heading errors in BMH at the ground truth markers. The $3 \sigma$ uncertainty includes the uncertainty of the ground truth (typically $<1^{\circ}$ ).

shaft and steering column. A separate trial was performed in which the vehicle was localized using the encoders and an LC, gyroscope, or scan matching (same gyroscope and scan matching algorithm as in the BMH experiments). The LC was given the a priori $\mathrm{AM} \phi=\left(1.3 \pm 2.5^{\circ}, 91.9 \pm 2.6^{\circ}\right)$ shown in Fig. 6d. A Novatel SPAN-SE-2 GPS receiver and CPT IMU, alongside commercial Novatel GPS/INS software were used to estimate the true two-dimensional pose.

The estimated paths of the vehicle and the ground truth path are shown in Fig. 10, and the heading errors are shown in Fig. 11. Because the estimated position is tightly coupled with the heading estimate, dead-reckoning position error growth in position is significantly arrested when using an LC. Regardless, position errors were introduced by imperfect encoder calibration, hills or uneven terrain, and local data association failures. In some cases, the uncertainty of the LC heading estimate was not properly represented. For example, the uncertainty of axis extraction combined with the uncertainty of the AM entries made data association difficult when surfaces in the environment were slightly misaligned (e.g., "poorly" parked cars), as was the case at $t=45 \mathrm{~s}$ in Fig. 11. Significant changes in heading in areas where no extractable axes existed in the environment also negatively affected heading estimation (e.g., at $t=215 \mathrm{~s}$ ). However, in both these cases, re-observation of entries in the a priori AM resulted in a quick recovery.

\section{CONCLUSION}

This paper introduces axis mapping, which estimates the axes of dominant surfaces in an environment given noisy LiDAR and gyroscope measurements. The problem is formulated as a large error minimization problem in a fashion similar to pose-graph SLAM; however, its unique properties (e.g., directional random variables, lack of positional information) merit a specialized solution. The algorithms are described in detail and were tested in several environments. It was found that accurate and efficient 2D axis mapping can be achieved in both structured (e.g., indoors) and unstructured (e.g., a mining drift) environments. Furthermore, the estimated axis maps were shown to be useful inputs for heading estimation using 


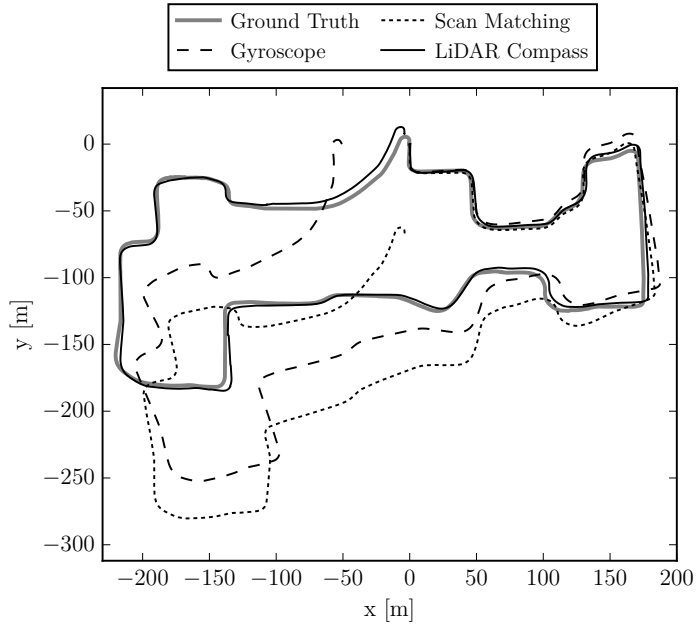

Fig. 10. The estimated $1.3 \mathrm{~km}$ paths of the robot for the Queen's University campus data set. The trial both started and ended at the origin.

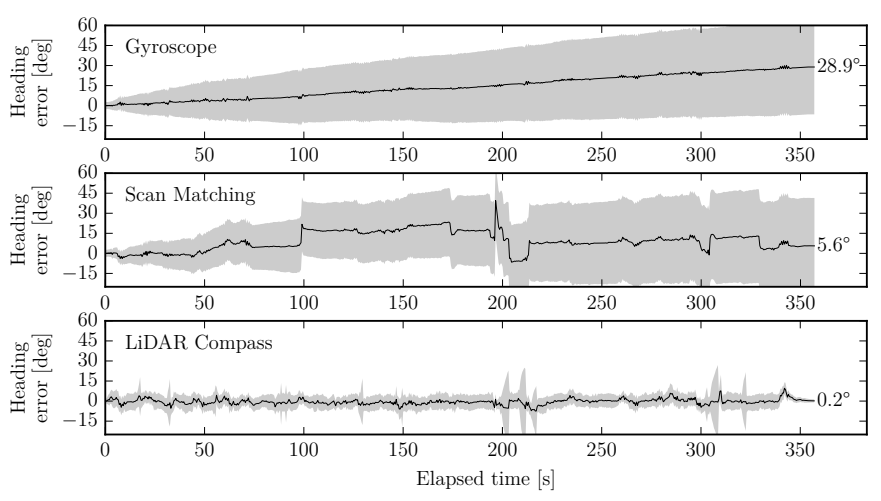

Fig. 11. The gyroscope, scan matching, and LC heading errors on the Queen's University campus. The $3 \sigma$ uncertainty includes the uncertainty of the ground truth (typically $<1.5^{\circ}$ ).

a LiDAR compass, resulting in bounded growth of heading errors when used as part of a localization algorithm. Work is underway to extend axis mapping to $3 \mathrm{D}$, which has application to the interpretation of rock faces for geotechnical purposes.

\section{ACKNOWLEDGMENT}

This project was funded by the Natural Sciences and Engineering Research Council of Canada under project 3979852011 and by the NSERC Canadian Field Robotics Network.

\section{REFERENCES}

[1] D. C. Wyllie and C. Mah, Rock Slope Engineering. New York, NY, USA: Spon Press, 2004.

[2] G. P. Giani, Rock Slope Stability Analysis. Rotterdam, Netherlands: A. A. Balkerma Publishers, 1992.

[3] G. Grisetti, R. Kümmerle, C. Stachniss, and W. Burgard, "A tutorial on graph-based SLAM," IEEE Intelligent Transportation Systems Magazine, vol. 2, no. 4, pp. 31-43, 2010.
[4] S. Thrun, W. Burgard, and D. Fox, Probabilistic Robotics. Cambridge, MA, USA: MIT Press, 2006.

[5] K. V. Mardia and P. E. Jupp, Directional Statistics. West Sussex, England: John Wiley \& Sons, Ltd., 2000.

[6] A. L. Gould, "A regression technique for angular variates," Biometrics, vol. 25 , no. 4, pp. 683-700, Dec. 1969.

[7] R. A. Johnson and T. E. Wehrly, "Some angular-linear distributions and related regression models," Journal of the American Statistical Association, vol. 73, no. 363, pp. 602-606, Sep. 1978.

[8] N. I. Fisher and A. J. Lee, "Regression models for an angular response," Biometrics, vol. 48, no. 3, pp. 665-677, Sep. 1992.

[9] Y. R. Sarma and S. R. Jammalamadaka, "Circular regression," in Statistical Sciences and Data Analysis: Proceedings of the Third Pacific Area Statistical Conference, Makuhari, Japan, Dec. 1991, pp. 109-128.

[10] U. Lund, "Least circular distance regression for directional data," Journal of Applied Statistics, vol. 26, no. 6, pp. 723-733, 1999.

[11] C. Hertzberg, R. Wagner, U. Frese, and L. Schröder, "Integrating generic sensor fusion algorithms with sound state representations through encapsulation of manifolds," Information Fusion, vol. 14, pp. 57-77, 2013.

[12] M. Azmani, S. Reboul, J.-B. Choquel, and M. Benjelloun, "A recursive fusion filter for angular data," in Proceedings of the IEEE International Conference on Robotics and Biomimetics, Guilin, China, Dec. 2009.

[13] G. Kurz, I. Gilitschenski, and U. D. Hanebeck, "Nonlinear measurement update for estimation of angular systems based on circular distributions," in Proceedings of the American Control Conference, Portland, OR, USA, Jun. 2014.

[14] L. Carlone, R. Aragues, J. A. Castellanos, and B. Bona, "A linear approximation for graph-based simultaneous localization and mapping," in Proceedings of the IEEE International Conference on Robotics: Science and Systems, Los Angeles, CA, USA, Jun. 2011.

[15] _ _ "A fast and accurate approximation for planar pose graph optimization," International Journal of Robotics Research, vol. 33, no. 7, pp. 965-987, Jun. 2014.

[16] W. E. Deming, Statistical Adjustment of Data. Mineola, New York: Dover Publications (New Edition), 2011.

[17] M. Ester, H.-P. Kriegel, J. Sander, and X. Xu, "A density-based algorithm for discovering clusters in large spatial databases with noise," in Proceedings of the 2nd International Conference on Knowledge Discovery and Data Mining. AAAI Press, 1996, pp. 226-231.

[18] J. Neira and J. Tardos, "Data association in stochastic mapping using the joint compatibility test," IEEE Transactions on Robotics and Automation, vol. 17, no. 6, pp. 890-897, Dec. 2001.

[19] G. Hu, K. Khosoussi, and S. Huang, "Towards a reliable SLAM backend," in Proceedings of the IEEE/RSJ International Conference on Intelligent Robots and Systems, 2013, pp. 37-43.

[20] G. Grisetti, C. Stachniss, and W. Burgard, "Nonlinear constraint network optimization for efficient map learning," IEEE Transactions on Intelligent Transportation Systems, vol. 10, no. 3, pp. 428-439, Sep. 2009.

[21] K. Konolige, G. Grisetti, R. Kümmerle, W. Burgard, B. Limketkai, and R. Vincent, "Efficient sparse pose adjustment for 2D mapping," in Proceedings of the IEEE/RSJ International Conference on Intelligent Robots and Systems, 2010, pp. 22-29.

[22] M. J. Gallant, J. A. Marshall, and B. K. Lynch, "Estimating the heading of a Husky mobile robot with a LiDAR compass based on direction maps," in Proceedings of the 10th International Conference on Intelligent Unmanned Systems, Montreal, Canada, Sep. 2014.

[23] I. Dryanovski and W. Morris, "Laser scan matcher," http://wiki.ros.org/ laser_scan_matcher, accessed: 2014-10-30.

[24] A. Censi, "An ICP variant using a point-to-line metric," in Proceedings of the IEEE International Conference on Robotics and Automation, Pasadena, CA, May 2008, pp. 19-25. 